\begin{tabular}{|c|c|}
\hline Title & Suppressing effective magnetic field and spin-relaxation rate by tuning barrier compositions in a (111) quantum well \\
\hline Author(s) & Iijima, Tomonori; Egami, Y oshiyuki; A kera, Hiroshi \\
\hline Citation & $\begin{array}{l}\text { Japanese Journal of A pplied Physics (JJA P), 59(10), } 100901 \\
\text { https://doi.org/10.35848/1347-4065/abb3d5 }\end{array}$ \\
\hline Issue Date & 2020-09-15 \\
\hline Doc URL & http:/hdl. handle.net/2115/82674 \\
\hline Rights & ( ) [2020] The Japan Society of A pplied Physics \\
\hline Type & article \\
\hline File Information & Spin_Relaxation_111_JJAP.pdf \\
\hline
\end{tabular}

Instructions for use 


\title{
Suppressing effective magnetic field and spin-relaxation rate by tuning barrier compositions in a (111) quantum well
}

\author{
Tomonori lijima $^{1}$, Yoshiyuki Egami², and Hiroshi Akera ${ }^{2}$ \\ ${ }^{1}$ Division of Applied Physics, Graduate School of Engineering, Hokkaido University, Sapporo, Hokkaido, \\ 060-8628, Japan \\ ${ }^{2}$ Division of Applied Physics, Faculty of Engineering, Hokkaido University, Sapporo, Hokkaido, 060-8628, \\ Japan
}

\begin{abstract}
Adjusting compositions of two barriers forming a (111)-oriented InGaAs/AIGaAsSb quantum well (QW) is proposed as a method to eliminate the effective magnetic field due to the Rashba and linear-in-momentum Dresselhaus spin-orbit interactions in the zeroth and first orders in the gate electric field. The resulting suppressed Dyakonov-Perel spin relaxation is used to achieve a high on/off ratio of the spin-relaxation rate at room temperature in a double-QW structure.
\end{abstract}

The effective magnetic field (EMF) due to the spin-orbit interaction (SOI) is employed to generate and manipulate the spin polarization in spintronics. ${ }^{1,2)}$ A strong EMF, which is preferable in creating and manipulating the spin polarization, has been achieved in semiconductor quantum wells (QWs). On the other hand, the EMF is the origin of the Dyakonov-Perel (DP) spin relaxation ${ }^{3-5}$ which reduces the magnitude of nonequilibrium spin polarization. Thus a weak EMF is desirable in order to maintain and transport the spin polarization. In this paper we propose a method to suppress the summed EMF caused by the Rashba ${ }^{6-9)}$ and linear-in-momentum Dresselhaus (linear Dresselhaus) ${ }^{5)}$ SOIs in a (111)-oriented QW and additionally design a double-QW structure which switches the EMF and the DP spin-relaxation rate by gate-voltage-induced electron transfer between two QWs.

Theoretical proposals to suppress the summed EMF and the resulting DP spin-relaxation rate by adjusting the gate electric field $E_{z}$ along the growth direction ( $z$ axis) have been made ${ }^{10-12)}$ in which the Rashba SOI proportional to $E_{z}$ is tuned to balance the linear Dresselhaus SOI in a (111)-oriented QW where these SOIs are the same function of the inplane momentum, $k_{x}$ and $k_{y}$, with different coefficients. This prediction has been subsequently confirmed by experiments. ${ }^{13-17)}$ Such suppression of the DP spin-relaxation in the (111) orientation has the advantage that it occurs for all three spin components, in contrast to that in 
$(001)^{18)}$ and $(110)^{5)}$ orientations $^{19)}$ where the spin relaxation of only one spin component is suppressed. However, from the point of view of the application to switching devices, ${ }^{20-23)}$ the suppression by tuning $E_{z}$ in the (111) orientation, as well as in (001) and (110) orientations, has a challenge to reduce the switching time for sufficiently suppressing the summed EMF since setting $E_{z}$ to the exact value at the balance needs a time much longer than the charging time constant of the gate. In addition the gate voltage, which gives the exact value of $E_{z}$ at the balance, is subject to unknown deviations in real samples of, for example, the dopant density from the designed value. Therefore it is desirable to suppress the summed EMF in a wide range of $E_{z}$ instead of a specific value of $E_{z}$.

In this paper we propose a method to suppress the sum of the Rashba EMF and the linear Dresselhaus EMF in a wide range of the gate electric field by extending the method proposed in our previous paper ${ }^{24)}$ to the presence of the linear Dresselhaus EMF. In our previous paper ${ }^{24)}$ we focused on the Rashba EMF in an $\operatorname{In}_{0.53} \mathrm{Ga}_{0.47} \mathrm{As} \mathrm{QW}$ formed by two $\mathrm{AlGaAsSb}$ barriers of the same composition. In such a symmetric QW the coefficient of the Rashba SOI vanishes at $E_{z}=0$ and is given by $\alpha=a_{\mathrm{so}} E_{z}$ except quite high $E_{z}$ where the proportionality constant $a_{\text {so }}$ is a function of valence-band offsets which vary with the Al fraction $x$ of the barrier compound $\mathrm{Al}_{x} \mathrm{Ga}_{1-x} \mathrm{As}_{y} \mathrm{Sb}_{1-y}$. We have shown in our previous paper ${ }^{24)}$ that, at a certain value of $x$, which is close to $0.3, a_{\mathrm{so}}$ vanishes and the Rashba SOI disappears at any value of $E_{z}$ (we have also demonstrated ${ }^{25}$ ) that the Rashba SOI can be eliminated by tuning a symmetric potential produced by the interface dipole ${ }^{26,27)}$ instead of adjusting the compound composition). In this paper we aim at suppressing, in a wide $E_{z}$ range, the total EMF consisting of the Rashba and linear Dresselhaus EMFs in a (111)-oriented QW, where the linear Dresselhaus EMF is the same, except the coefficient, as the Rashba EMF. Since the linear Dresselhaus EMF is nonzero at $E_{z}=0$, the Rashba EMF needs to have a term of the zeroth order in $E_{z}$ to balance the linear Dresselhaus EMF. To realize such Rashba EMF, in this paper, we introduce an asymmetry in the $\mathrm{Al}$ fraction between the left and right barriers [see Fig. 1(a)] and eliminate the summed EMF in the zeroth and first orders in $E_{z}$ by adjusting the $\mathrm{Al}$ fractions of the two barriers.

In addition we demonstrate the switching of the summed EMF in a double-QW structure [Fig. 2(a)] with use of the gate-voltage-induced electron transfer between the abovementioned QW, designed to suppress the summed EMF, and another QW with a strong EMF, by extending the Rashba EMF switching in our previous paper. ${ }^{28)} \mathrm{A}$ stronger EMF is obtained by narrowing an asymmetric QW. ${ }^{29)}$ We show in such a double-QW structure that the room-temperature on/off ratio of the spin-relaxation rate in the DP mechanism due to the 
(a)

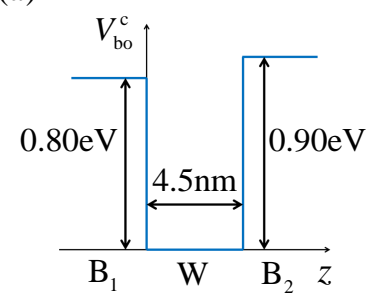

(b)

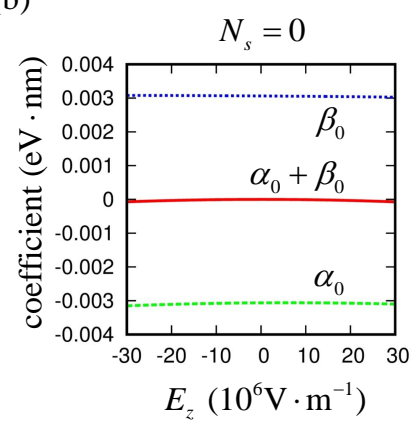

Fig. 1. (a) Potential due to the conduction-band offset $V_{\mathrm{bo}}^{\mathrm{c}}(z)$ in a (111)-oriented QW with the barrier-composition asymmtery. The well material $\mathrm{W}$ is $\operatorname{In}_{0.53} \mathrm{Ga}_{0.47} \mathrm{As}$ and the barrier material is $\mathrm{Al}_{x} \mathrm{Ga}_{1-x} \mathrm{As}_{y} \mathrm{Sb}_{1-y}$ with the barrier height $(\mathrm{eV})$ of $0.436+1.43 x .^{29)}$ The Al fraction $x$ is $x_{1}=0.253$ $\left(x_{2}=0.323\right)$ for the barrier material $\mathrm{B}_{1}\left(\mathrm{~B}_{2}\right)$. (b) The coefficient of the Rashba SOI for an electron in the ground subband $\alpha_{0}$ (dashed line), that of the Dresselhaus SOI $\beta_{0}$ (dotted line), and $\alpha_{0}+\beta_{0}$ (solid line), in which the subscript " 0 " denotes the ground subband, are plotted as a function of the applied electric field $E_{z}$ perpendicular to the QW at the electron sheet density $N_{s}=0$.

Rashba and linear Dresselhaus SOIs reaches $10^{7}$ even when $E_{z}$ in the off state deviates from the optimum value, which gives the lowest spin-relaxation rate, by $10 \%$ of the difference in $E_{z}$ between on and off states. In calculating the spin-relaxation rate we employ a formula for the multi-subband case which we have derived ${ }^{28)}$ by extending the Dyakonov-Perel formula ${ }^{3-5)}$ for a single band (subband). Finally we discuss the influence of the spin relaxation caused by the cubic Dresselhaus SOI.

The Hamiltonian $H$ for a conduction-band electron confined in a QW structure consists of $H_{\mathrm{QW}}$ describing the motion in the QW potential, $H_{\mathrm{so}}$ the SOI, and $V_{\text {imp }}$ the impurity potential:

$$
H=H_{\mathrm{QW}}+H_{\mathrm{so}}+V_{\mathrm{imp}}
$$

$H_{\mathrm{QW}}$ is given by

$$
H_{\mathrm{QW}}=\frac{\hat{\boldsymbol{p}}^{2}}{2 m}+V_{\mathrm{W}}(z)
$$

where $\hat{\boldsymbol{p}}=\left(\hat{p}_{x}, \hat{p}_{y}, \hat{p}_{z}\right)=-i \hbar \boldsymbol{\nabla}=-i \hbar\left(\nabla_{x}, \nabla_{y}, \nabla_{z}\right)$ and $m$ is the effective mass of the conduction band. The potential $V_{\mathrm{W}}(z)$ is the sum of $V_{\mathrm{bo}}^{\mathrm{c}}(z)$ the potential due to conduction-band offsets at heterointerfaces and $V_{\mathrm{es}}(z)$ the electrostatic potential due to donors outside of the QW structure and the applied electric field $E_{z}$ as well as the self-consistently-calculated Hartree 
(a)

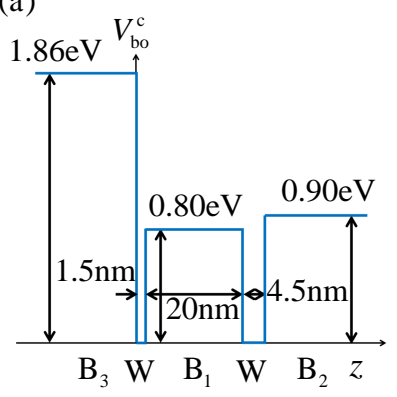

(b)

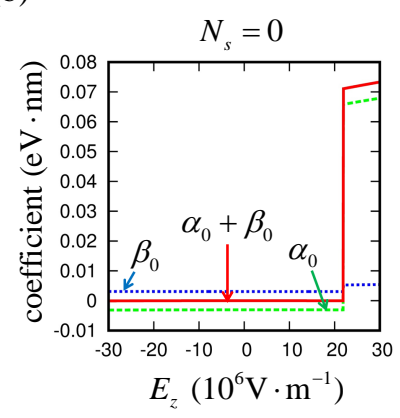

Fig. 2. (a) Potential due to the conduction-band offset $V_{\mathrm{bo}}^{\mathrm{c}}(z)$ in a (111)-oriented double-QW structure consisting of the QW (right) with the EMF suppressed as shown in Fig. 1(b) and another QW (left) with a strong EMF. Materials W, $\mathrm{B}_{1}$, and $\mathrm{B}_{2}$ are the same as in Fig. 1. The barrier material $\mathrm{B}_{3}$ is $\mathrm{AlAs}_{y} \mathrm{Sb}_{1-y}$. (b) The same coefficients as in Fig. 1(b) are plotted for the double-QW structure. The sum $\alpha_{0}+\beta_{0}$ increases discontinuously around $E_{z}=20 \times 10^{6} \mathrm{~V} \cdot \mathrm{m}^{-1}$ because $\alpha_{0}+\beta_{0}$ is negligible in the lower $E_{z}$ side where the ground-subband wave function $|0\rangle$ is in the right QW [see Fig.1(b)], while it becomes large in the higher $E_{z}$ side where $|0\rangle$ moves to the left QW with the lower potential energy at such values of $E_{z}$. The left QW has a large $\alpha_{0}$ because it is strongly asymmetric in barrier composition.

potential:

$$
V_{\mathrm{W}}(z)=V_{\mathrm{bo}}^{\mathrm{c}}(z)+V_{\mathrm{es}}(z)
$$

Applying the periodic boundary conditions in the $x$ and $y$ directions, each eigenstate of $H_{\mathrm{QW}}$ is labelled by the wave vector in the $x y$ plane, $\boldsymbol{k}=\left(k_{x}, k_{y}\right)$, the subband index, $n(=0,1,2, \cdots)$, and the $z$ component of spin, $\sigma(=\uparrow, \downarrow)$. The corresponding eigenenergy and eigenvector are denoted by $\varepsilon_{n \boldsymbol{k} \sigma}$ and $|n \boldsymbol{k} \sigma\rangle$ :

$$
H_{\mathrm{QW}}|n \boldsymbol{k} \sigma\rangle=\varepsilon_{n \boldsymbol{k} \sigma}|n \boldsymbol{k} \sigma\rangle
$$

where

$$
\varepsilon_{n \boldsymbol{k} \sigma}=\varepsilon_{n}+\hbar^{2} k^{2} / 2 m, \quad|n \boldsymbol{k} \sigma\rangle=|n\rangle|\boldsymbol{k}\rangle|\sigma\rangle,
$$

with $k=|\boldsymbol{k}|$. Here $\varepsilon_{n}$ and $|n\rangle$ are the eigenenergy and eigenvector of the Hamiltonian associated with the motion along the $z$ direction:

$$
\left[\frac{\hat{p}_{z}^{2}}{2 m}+V_{\mathrm{W}}(z)\right]|n\rangle=\varepsilon_{n}|n\rangle
$$

We solve the Schrödinger equation [Eq. (6)] for $\varepsilon_{n}$ and $|n\rangle$ and the Poisson equation for $V_{\mathrm{es}}(z)$ self-consistently. The applied electric field $E_{z}$ and the donor density are taken into account in the boundary condition for the Poisson equation. The eigenvector $|n\rangle$ for each subband is 
used to obtain the subband-dependent coefficients of the Rashba and Dresselhaus SOIs.

The SOI $H_{\mathrm{so}}$ consists of the Rashba SOI and the linear Dresselhaus SOI. $H_{\text {so }}$ conserves $\boldsymbol{k}$. We neglect intersubband processes induced by $\left\langle n \boldsymbol{k}\left|H_{\mathrm{so}}\right| n^{\prime} \boldsymbol{k}\right\rangle\left(n \neq n^{\prime}\right)$ by restricting our discussion to the subband structure with large energy separations $\left|\varepsilon_{n}-\varepsilon_{n^{\prime}}\right|$. Diagonal matrix elements $\left\langle n \boldsymbol{k}\left|H_{\mathrm{so}}\right| n \boldsymbol{k}\right\rangle$ in (111)-oriented QW structures are given by

$$
\left\langle n \boldsymbol{k}\left|H_{\mathrm{so}}\right| n \boldsymbol{k}\right\rangle=\left(\alpha_{n}+\beta_{n}\right)\left(\sigma_{x} k_{y}-\sigma_{y} k_{x}\right),
$$

with $\sigma_{x}$ and $\sigma_{y}$ the Pauli spin operators. Here $\alpha_{n}$ and $\beta_{n}$ are the subband-dependent coefficients of the Rashba SOI and the linear Dresselhaus SOI, respectively. The formula for $\alpha_{n}$ expressed by valence-band offsets and $V_{\mathrm{es}}(z)$ is given in our previous papers ${ }^{24,28,29)}$ based on the 8-band $\boldsymbol{k} \cdot \boldsymbol{p}$ theory for heterostructures. ${ }^{30-32)}$ The coefficient $\beta_{n}$ is given by

$$
\beta_{n}=\frac{2}{\sqrt{3}} \gamma\left\langle n\left|\hat{k}_{z}^{2}\right| n\right\rangle,
$$

with $\hat{k}_{z}=\hat{p}_{z} / \hbar$. Here we have neglected the difference in the coefficient $\gamma$ of the Dresselhaus $\mathrm{SOI}^{33)}$ between well and barrier materials and use the value of $\gamma$ for the well material $\operatorname{In}_{0.53} \mathrm{Ga}_{0.47} \mathrm{As}$. The formula for evaluating the value of $\gamma$ is expressed ${ }^{34)}$ by the parameters in the 14-band $\boldsymbol{k} \cdot \boldsymbol{p}$ theory.

The impurity potential $V_{\mathrm{imp}}(\boldsymbol{r})$ with $\boldsymbol{r}=(x, y, z)$ is the potential due to impurities which are distributed uniformly and randomly in the QW structure. We assume that $V_{\text {imp }}(\boldsymbol{r})$ is the sum of potentials due to each impurity.

The SOI in the form of Eq. (7) induces the following spin relaxation: ${ }^{5)}$

$$
\frac{d S_{v}}{d t}=-\frac{S_{v}}{\tau_{v v}}(v=x, y, z), \quad \tau_{x x}=\tau_{y y}=2 \tau_{z z},
$$

where $\tau_{v v}$ is the spin-relaxation time of the spin polarization in the $v$ direction, $S_{v}$. We calculate $\tau_{z z}$ with use of the formula derived for the multi-subband case. ${ }^{28)}$ In the derivation of the formula, the following approximations are employed. (1) Terms up to the second order in $V_{\text {imp }}(\boldsymbol{r})$ are retained. (2) The average over the $x$ and $y$ coordinates of each impurity is taken. (3) According to the Dyakonov-Perel theory, ${ }^{3-5)}$ the SOI is neglected in the collision term of the kinetic equation for the density matrix, and terms up to the first order in $\Omega_{n} \tau_{n}$, with $\Omega_{n}=(2 / \hbar)\left(\alpha_{n}+\beta_{n}\right) k$ and $\tau_{n}$ the momentum relaxation time, are retained in solving for the density matrix. The density matrix of the zeroth order in $\Omega_{n} \tau_{n}$ is described by the Fermi-Dirac distribution with the temperature $T$ and the $\sigma$-dependent chemical potential, $\mu_{\sigma}$. (4) The limit of $\left|\mu_{\uparrow}-\mu_{\downarrow}\right| \rightarrow 0$ is taken in deriving the formula for $\tau_{z z}$.

In evaluating the spin-relaxation rate, the potential induced by an impurity at $\boldsymbol{r}_{\text {imp }}$ is modeled by the short-range potential: $u_{0} \delta\left(\boldsymbol{r}-\boldsymbol{r}_{\text {imp }}\right)$. With use of the potential strength $u_{0}$ we 
introduce units of the momentum-relaxation time and the spin-relaxation time, $\tau_{p 0}$ and $\tau_{s 0}$, which are defined by

$$
\begin{aligned}
\frac{1}{\tau_{p 0}} & =n_{\mathrm{imp}} u_{0}^{2} \frac{m}{\hbar^{3} a_{B}^{*}}, \\
\frac{1}{\tau_{s 0}} & =10^{6} \frac{\eta^{2}\left(\mathrm{Ry}^{*}\right)^{2}}{\hbar^{2}\left(a_{B}^{*}\right)^{4}} \tau_{p 0}=10^{6} \frac{\eta^{2} \hbar\left(\mathrm{Ry}^{*}\right)^{2}}{n_{\mathrm{imp}} u_{0}^{2} m\left(a_{B}^{*}\right)^{3}},
\end{aligned}
$$

respectively with $n_{\text {imp }}$ the impurity volume density and $\eta$ the effective SOI constant of the conduction band. ${ }^{24,28,29)}$ Here $a_{B}^{*}=\hbar^{2} \epsilon /\left(m e^{2}\right)$ and $\mathrm{Ry}^{*}=\hbar^{2}\left(a_{B}^{*}\right)^{-2} /(2 m)$ with $\epsilon$ the static dielectric constant. We use material parameters for the well material $\operatorname{In}_{0.53} \mathrm{Ga}_{0.47} \mathrm{As}$ in which $a_{B}^{*}=17.1 \mathrm{~nm}$ and $\mathrm{Ry}^{*}=3.04 \mathrm{meV}$.

First we consider a QW in Fig. 1(a). The well material $\mathrm{W}$ is $\operatorname{In}_{0.53} \mathrm{Ga}_{0.47} \mathrm{As}$ and the barrier material is $\mathrm{Al}_{x} \mathrm{Ga}_{1-x} \mathrm{As}_{y} \mathrm{Sb}_{1-y}$ in which the $\mathrm{Al}$ fraction $x$ is $x_{1}=0.253$ for $\mathrm{B}_{1}$ and $x_{2}=0.323$ for $\mathrm{B}_{2}$. The values of $x_{1}$ and $x_{2}$ are determined to eliminate the sum of the Rashba coefficient in the ground subband $\alpha_{0}$ and the corresponding Dresselhaus coefficient $\beta_{0}$ in the zeroth and first orders in $E_{z}$ at the vanishing electron sheet density, $N_{s}=0$ (the fraction of As, $y$, is determined so that the layer is lattice matched to InP). As shown in Fig. 1(b), $\alpha_{0}+\beta_{0}$ is suppressed in a wide range of $E_{z}$ to a value much smaller than $\beta_{0}$.

Next we consider a double-QW structure in Fig. 2(a), which consists of a QW (right) with the suppressed EMF and another QW (left) which is made highly asymmetric (the Al fraction of $\mathrm{B}_{3}$ is $x_{3}=1$ ) and narrow to acquire a strong Rashba EMF even at $E_{z}=0 .{ }^{29)}$ The large separation between the QWs substantially reduces the mixing of wave functions belonging to the left and right QWs in the $E_{z}$ region where the spin relaxation is on or off. We demonstrate the $E_{z}$-induced switching of $\alpha_{0}+\beta_{0}$ in Fig. 2(b): $\alpha_{0}+\beta_{0}$ is negligibly small in the off state at $E_{z} \sim 0$ with an electron localized in the right QW with the suppressed EMF, while it acquires a large value in the large $E_{z}$ region where the electron transfers to the left QW and is subject to the Rashba EMF much stronger than $\beta_{0}$.

In Fig. 3 we present the $E_{z}$-induced switching of the spin-relaxation rate $1 / \tau_{z z}$ in linear scale [Fig. 3(a)] and logarithmic scale [Fig. 3(b)] in the double-QW structure. When the temperature increases from absolute zero to room temperature, Fig. 3(b) shows that, $1 / \tau_{z z}$ increases by orders of magnitude in the range of $0<E_{z} /\left(10^{6} \mathrm{~V} \cdot \mathrm{m}^{-1}\right)<20$ because the increased electron population in excited subbands $(n \geq 1)$ causes undesired spin relaxation. However, the on/off ratio of $1 / \tau_{z z}$, when on and off values of $1 / \tau_{z z}$ are taken at $E_{z} /\left(10^{6} \mathrm{~V} \cdot \mathrm{m}^{-1}\right)=30$ and -5 , respectively, reaches the order of $10^{7}$ from absolute zero to room temperature since we successfully increase energy separations from the ground subband to such excited subbands 
(a)

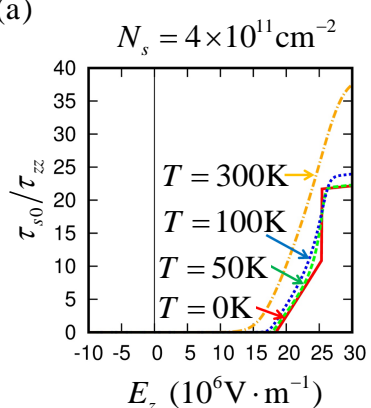

(b)

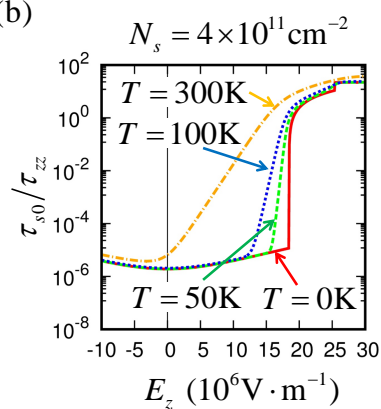

Fig. 3. Temperature dependence of the spin-relaxation rate $1 / \tau_{z z}$ in the double-QW structure in Fig. 2(a) as a function of the perpendicular electric field $E_{z} . N_{s}$ is the electron sheet density. Plotted in (a) linear scale and (b) semi-logarithmic scale.

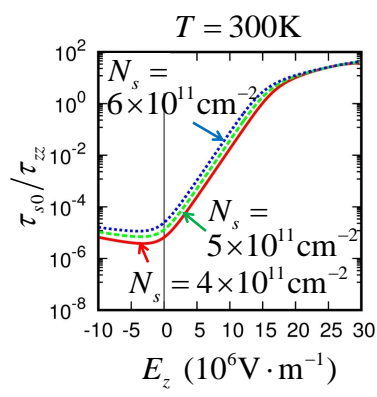

Fig. 4. Electron sheet density $N_{s}$ dependence of the spin-relaxation rate $1 / \tau_{z z}$ in the double-QW structure in Fig. 2(a) as a function of the perpendicular electric field $E_{z}$ at $T=300 \mathrm{~K}$.

in the off region by making the left QW narrower than the right $\mathrm{QW}$. The dependence on the electron sheet density $N_{s}$ of $1 / \tau_{z z}$ at room temperature is not strong as presented in Fig. 4.

In Fig. 5 we present the switching of the spin-relaxation rate $1 / \tau_{z z}$ in another double-QW structure with the barrier material $B_{3}$ in the right side. The on value of $1 / \tau_{z z}$ is smaller than the double-QW structure with $\mathrm{B}_{3}$ in the left side since the direction of the Rashba EMF is opposite to that of the linear Dresselhaus EMF in the on state. However the on/off ratio of $1 / \tau_{z z}$ has the same order of magnitude in both double-QW structures.

Finally we discuss the influence of the cubic Dresselhaus SOI ${ }^{10,35)}$ which is not taken into account in the calculation of this work. Since the cubic Dresselhaus SOI is of the third order in the inplane momentum $k=|\boldsymbol{k}|$, it is smaller than the linear Dresselhaus SOI by $(k d / \pi)^{2}$ with $d$ the well width and thus was not considered in most studies on narrow QWs. ${ }^{536)}$ However, in the previous studies ${ }^{10-17)}$ and the present study, the linear Dresselhaus SOI and the Rashba 
(a)

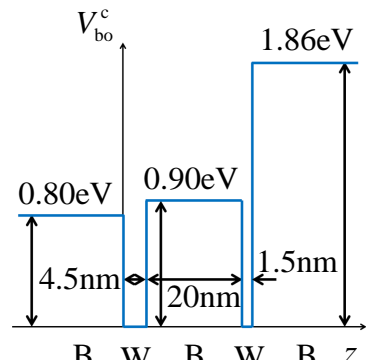

(b)

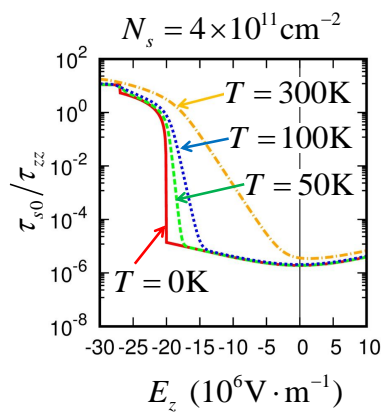

Fig. 5. (a) Potential due to the conduction-band offset $V_{\mathrm{bo}}^{\mathrm{c}}(z)$ in another double-QW structure in which the location of the barrier material $B_{3}$ is different from that in Fig. 2(a). (b) The temperature dependence of the spin-relaxation rate $1 / \tau_{z z}$ as a function of the perpendicular electric field $E_{z}$.

SOI is balanced in the off state. Therefore the cubic Dresselhaus SOI is dominant in the off state, while it is negligible in the on state. We estimate the off value of the spin-relaxation rate due to the cubic Dresselhaus SOI and find that the on/off ratio of the spin-relaxation rate in the double-QW structures [Figs. 2(a) and 5(a)] at $N_{s}=4 \times 10^{11} \mathrm{~cm}^{-2}$ reduces to $10^{5}$ at absolute zero and $10^{4}$ at room temperature when the cubic Dresselhaus SOI is taken into account.

In conclusion, we have shown that, in a (111)-oriented InGaAs/AlGaAsSb QW, the sum of the Rashba EMF and the linear Dresselhaus EMF can be eliminated in both the zeroth and the first orders in the gate electric field by adjusting the $\mathrm{Al}$ fractions of two materials used as the left and right barriers. We have demonstrated, in a double-QW structure consisting of the QW with the suppressed EMF and another QW with a strong EMF, that the room-temperature spin-relaxation rate in the DP mechanism is switched with a high on/off ratio by changing the gate electric field.

This work was supported by Grant-in-Aid for Scientific Research (C) Grant No. JP17K05484 from the Japan Society for the Promotion of Science (JSPS). 


\section{References}

1) I. Žutić, J. Fabian, and S. D. Sarma, Rev. Mod. Phys. 76, 323 (2004).

2) D. D. Awschalom and M. E. Flatté, Nat. Phys. 3, 153 (2007).

3) M. I. Dyakonov and V. I. Perel, Sov. Phys. JETP 33, 1053 (1971).

4) M. I. Dyakonov and V. I. Perel, Sov. Phys. Solid State 13, 3023 (1972).

5) M. I. Dyakonov and V. Y. Kachorovskii, Sov. Phys. Semicond. 20, 110 (1986).

6) E. I. Rashba, Sov. Phys. Solid State 2, 1109 (1960).

7) F. J. Ohkawa and Y. Uemura, J. Phys. Soc. Jpn. 37, 1325 (1974).

8) Y. A. Bychkov and E. I. Rashba, J. Phys. C 17, 6039 (1984).

9) Y. A. Bychkov and E. I. Rashba, JETP Lett. 39, 78 (1984).

10) X. Cartoixà, D. Z.-Y. Ting, and Y.-C. Chang, Phys. Rev. B 71, 045313 (2005).

11) I. Vurgaftman and J. R. Meyer, J. Appl. Phys. 97, 053707 (2005).

12) B. Y. Sun, P. Zhang, and M. W. Wu, J. Appl. Phys. 108, 093709 (2010).

13) A. Balocchi, Q. H. Duong, P. Renucci, B. L. Liu, C. Fontaine, T. Amand, D. Lagarde, and X. Marie, Phys. Rev. Lett. 107, 136604 (2011).

14) H. Q. Ye, G. Wang, B. L. Liu, Z. W. Shi, W. X. Wang, C. Fontaine, A. Balocchi, T. Amand, D. Lagarde, P. Renucci, and X. Marie, Appl. Phys. Lett. 101, 032104 (2012).

15) K. Biermann, A. Hernández-Mínguez, R. Hey, and P. V. Santos, J. Appl. Phys. 112, 083913 (2012).

16) A. Hernández-Mínguez, K. Biermann, R. Hey, and P. V. Santos, Phys. Rev. Lett. 109, 266602 (2012).

17) G. Wang, A. Balocchi, D. Lagarde, C. R. Zhu, T. Amand, P. Renucci, Z. W. Shi, W. X. Wang, B. L. Liu, and X. Marie, Appl. Phys. Lett. 102, 242408 (2013).

18) N. S. Averkiev and L. E. Golub, Phys. Rev. B 60, 15582 (1999).

19) The persistent spin helix (see, for a review, Ref. 36) occurs in the same condition as the suppression of the DP spin relaxation. They occur at $\alpha_{0}= \pm \beta_{0}$ in the (001) orientation ${ }^{18,36)}$ with $\alpha_{0}$ and $\beta_{0}$ the coefficient of the Rashba SOI and that of the linear Dresselhaus SOI in the ground subband as well as at $\alpha_{0}=0$ in the (110) orientation. ${ }^{5,36)}$

20) J. Schliemann, J. C. Egues, and D. Loss, Phys. Rev. Lett. 90, 146801 (2003).

21) X. Cartoixà, D. Z.-Y. Ting, and Y.-C. Chang, Appl. Phys. Lett. 83, 1462 (2003).

22) K. C. Hall, W. H. Lau, K. Gündoğdu, M. E. Flatté, and T. F. Boggess, Appl. Phys. Lett. 83, 2937 (2003).

23) K. C. Hall and M. E. Flatté, Appl. Phys. Lett. 88, 162503 (2006). 
24) H. Akera, H. Suzuura, and Y. Egami, Phys. Rev. B 92, 205311 (2015).

25) Y. Egami and H. Akera, Appl. Phys. Express 10, 063007 (2017).

26) F. Capasso, A. Y. Cho, K. Mohammed, and P. W. Foy, Appl. Phys. Lett. 46, 664 (1985).

27) S. Modesti, D. Furlanetto, M. Piccin, S. Rubini, and A. Franciosi, Appl. Phys. Lett. 82, 1932 (2003).

28) T. Iijima and H. Akera, Phys. Rev. Applied 13, 064075 (2020).

29) H. Akera, H. Suzuura, and Y. Egami, Phys. Rev. B 95, 045301 (2017).

30) R. Lassnig, Phys. Rev. B 31, 8076 (1985).

31) E. Bernardes, J. Schliemann, M. Lee, J. C. Egues, and D. Loss, Phys. Rev. Lett. 99, 076603 (2007).

32) R. S. Calsaverini, E. Bernardes, J. C. Egues, and D. Loss, Phys. Rev. B 78, 155313 (2008).

33) G. Dresselhaus, Phys. Rev. 100, 580 (1955).

34) J.-M. Jancu, R. Scholz, E. A. de Andrada e Silva, and G. C. La Rocca, Phys. Rev. B 72, 193201 (2005).

35) X. Cartoixà, L.-W. Wang, D.-Y. Ting, and Y.-C. Chang, Phys. Rev. B 73, 205341 (2006).

36) J. Schliemann, Rev. Mod. Phys. 89, 011001 (2017). 